\title{
Coverage of CFS within U.S. Medical Schools
}

\author{
T. Mark Peterson, D.D.S, Thomas W. Peterson, D.D.S., Sarah Emerson, B.S., Eric Regalbuto, Meredyth A. \\ Evans, M.A..", Leonard A. Jason, Ph.D. \\ Center for Community Research, DePaul University, Chicago, 60614, Illinois, United States \\ *Corresponding Author: mevans24@depaul.edu
}

Copyright $@ 2013$ Horizon Research Publishing All rights reserved.

\begin{abstract}
Little is known about the extent to which chronic fatigue syndrome (CFS) is covered within medical schools in the United States (U.S.) The current study is an exploration of the extent that CFS is covered in the areas of treatment, research, and curricula in U.S. Medical Schools. Surveys were sent to personnel at 132 accredited U.S. medical schools and a total of 71 schools responded. The extent of coverage across the three domains was extremely limited. Only $29.6 \%$ of schools met the clinical criterion, $28.2 \%$ met the curricula criterion, and $15 \%$ met the research criterion. Only four of the $71(5.6 \%)$ responding schools met criteria for all three domains. While the current study is preliminary, it points to significant gaps in the coverage of CFS among medical institutions, which is likely impacting the ability of physicians to fully acknowledge, understand, effectively treat, and find a cure for CFS.
\end{abstract}

Keywords Chronic Fatigue Syndrome, Coverage, U.S. Medical Schools

\section{Introduction}

Chronic fatigue syndrome (CFS) is defined by the Fukuda et al. (1994) [1] case criteria as an illness involving six or more months of chronic fatigue that causes considerable reductions in an individual's work, social, and personal activities. Furthermore, the Fukuda criteria are polythetic, as they consist of a set of symptoms in which not all need to be present to make a diagnosis. Specifically, an individual with CFS must have four out of eight accompanying symptoms (e.g. sore throat, lymph node pain, muscle pain, joint pain, post-exertional malaise, headaches of a new or different type, memory and concentration difficulties, and unrefreshing sleep) that have persisted for at least six months or longer [1].

Over the past decade, with increasing dissatisfaction with the Fukuda et al. [1] criteria, two new case definitions have emerged, with one called Myalgic Encephalomyelitis (ME/CFS) and the other Myalgic Encephalomyelitis (ME) $[2,3]$. They differ from the polythetic Fukuda et al. (1994) criteria [1], as they require the presence of cardinal symptoms including post exertional malaise (PEM), neurocognitive problems, and sleep difficulties. Early prevalence estimates for CFS published by the Centers for Disease Control and Prevention (CDC), ranged from .002\% to $.0073 \%$ [4]. Following these initial estimates, Jason et al. [5] utilized a randomized community-based sample and found that approximately $.42 \%$ of the sample met criteria for CFS. Another study conducted by the CDC also utilized a community-based sample and estimated a prevalence rate of $.24 \%$. More recent estimates published by the CDC published an estimated prevalence rate of $2.54 \%$ [6], which was approximately 10 times higher than previous CDC estimates [7]. These estimates were based off of the controversial empirical case criteria [8] that have been criticized for their inability to discriminate those with CFS from those with a primary mood disorder [9]. There is growing consensus that as many as 800,000 to $1,000,000$ individuals are affected by this illness in the U.S.

Despite this substantial number of individuals living with this highly debilitating illness, there is a lack of information regarding the extent that either $\mathrm{CFS}, \mathrm{ME} / \mathrm{CFS}$ or $\mathrm{ME}$ are taught, researched, and clinically treated within medical schools in the U.S. A recent study by Jason et al. [10] reviewed 119 medical textbooks from a variety of medical specialties and found that CFS, ME, and ME/CFS were largely underrepresented, and of the total pages reviewed, only .09 percent had content on these illnesses. Yet, illnesses with lower prevalence rates, such as multiple sclerosis and Lyme disease had considerably more content. According to Rabow et al. [10] medical textbooks provide a key foundation in the training of medical staff and students. Specifically, they provide references and reviews, which include important information regarding best practices for conceptualizing, diagnosing, and treating various health conditions and illnesses. This underrepresentation in medical textbooks may suggest a potential gap in the degree of information on CFS, ME/CFS, and ME that is covered within medical classrooms across the country. Without adequate resources and education about these complex illnesses, health care professionals will not have the necessary information and training to understand, diagnose, and treat them effectively.

The purpose of the current exploratory study was to determine the degree to which CFS is studied, taught, and 
treated at medical schools in the U.S. Medical schools' coverage of CFS was assessed based on the following criteria: clinical treatment of CFS, research pertaining to CFS, and CFS-specific curricula currently taking place at and being used by medical schools in the U.S. There is no published information on the extent to which medical schools include this type of content within their teaching, research, and treatment centers.

\section{Materials and Methods}

The initial sample consisted of 132 medical schools in the U.S., all accredited by the Association of American Medical Colleges (AAMC). The names of the 132 schools were retrieved from the list of AAMC schools accredited in 2010. Out of the 132 schools that were initially identified, a total of 71 (54\%) schools participated. Twenty-four schools (34\%) that responded are located in the Northeast, $10(14 \%)$ are located in the Southeast, 24 (34\%) are located in the Midwest, and $13(18 \%)$ are located in the Western region of the United States.

The survey questions investigated three different domains: clinical treatment of CFS, research on CFS, and medical school curricula covering CFS. The surveys were sent out to 132 schools. For the first domain, school representatives were asked to identify whether clinical treatment for CFS was offered, and if yes, which (if any) faculty members were considered experts in treating CFS. For the second domain, schools were asked to identify any significant CFS research (current projects or papers published) taking place within the confines of the school. Lastly, for the third domain, a question on the survey requested information concerning the extent of CFS coverage and instruction within the medical schools' curricula.

Surveys were sent out to the president of each medical institution, and schools that did not respond or that experienced a change in presidency were re-sent the initial study invitation and survey five months later. The majority of the schools that responded did so in written format, and three schools responded to the survey over the phone.

After responses were returned from participating schools, they were rated on each study criterion (e.g., treatment, research, and medical curricula). Responses indicating the presence of faculty experts in CFS or the existence of CFS treatments offered at the institution were determined as meeting the treatment criterion. Responses indicating any current or recent CFS related research efforts were documented as meeting the research criterion. Responses indicating CFS-specific instruction related to the history, understanding, symptomology, diagnosis, and/or treatment of CFS were documented as meeting the curricula criterion. In order to ensure reliability, two researchers assessed school responses independently. Excellent inter-rater reliability was achieved with a Cohen's kappa score of 0.90 for the curricula domain, 0.96 for the treatment domain, and 0.99 for the research domain.

\section{Results}

Of the 71 medical schools that participated in the current study, $21(29.6 \%)$ met the clinical treatment criterion (see Table 1). Of the 21 schools that met this criterion, five $(23.8 \%)$ reported that treatment was administered by faculty from the rheumatologic department, and one school (4.8\%) indicated a specific specialty in treating pediatric areas. In regard to the research criterion, $11(15.5 \%)$ schools indicated that CFS-related research projects and/or publications were taking place at their institutions (see Table 1). Lastly, 20 $(28.2 \%)$ indicated that information on CFS was included within the medical curricula (see Table 1). Also noteworthy, only four of the $71(5.6 \%)$ responding schools met criteria for all three domains, $10(14.2 \%)$ schools met criteria for two of the three domains, and $57(80.2 \%)$ of the responding schools met criteria for only one domain.

Table 1. Percentage of medical schools by geographic region covering CFS within treatment centers, research, and school curricula

\begin{tabular}{|c|c|c|c|}
\hline Region & Treatment & Research & Curriculum \\
\hline Northeast & $8 / 24(29.2 \%)$ & $5 / 24(20.8 \%)$ & $5 / 24(20.8 \%)$ \\
\hline Southeast & $0 / 10(0.0 \%)$ & $0 / 10(0.0 \%)$ & $3 / 10(30.0 \%)$ \\
\hline Midwest & $10 / 24(41.7 \%)$ & $4 / 24(16.6 \%)$ & $7 / 24(29.2 \%)$ \\
\hline West & $3 / 13(23.1 \%)$ & $2 / 13(15.4 \%)$ & $5 / 13(38.5 \%)$ \\
\hline
\end{tabular}

\section{Discussion}

This exploratory study investigated the extent to which CFS-related treatment, research, and instruction takes place within U.S. medical schools. The results of the current study revealed that the degree of CFS coverage in medical schools is extremely limited. Overall, a higher number of schools met the clinical and curricula criteria $(29.6 \%$ and $28.2 \%$, respectively) compared to the research criterion (15.5\%). Very few schools qualified for all three criteria $(5.6 \%)$. It is also important to note that of the 21 schools that met the treatment criterion, the majority of schools reported that there were only one or two physicians treating CFS, and 11 total schools reported having a physician or physicians with a specialty or expertise in treating CFS. The majority of physicians treating CFS at U.S. Medical Schools were also primary care physicians.

Only 71 of 132 medical schools responded to the study survey; therefore, it is possible that these results do not reflect the most accurate estimate of CFS coverage in U.S. medical schools. There was a considerable effort to reach unresponsive schools (i.e., schools were re-sent study surveys after five months, and presidents were re-contacted when there was a known change in leadership), and it is likely that these schools did not have much CFS coverage. Another limitation of this investigation is the possible likelihood of social desirability bias. The surveys were initially sent to the schools by a family member of an 
afflicted individual, and this was clearly stated in the introduction letter that was sent with each survey. The respondents at each school may have felt compelled to increase their CFS-related activity in order to present their institution in a more favorable light.

The current study utilized broad criteria to determine whether U.S. medical schools covered CFS in the domains of treatment, research, and curricula. Future studies of this topic might include more narrowly defined criteria in order to determine which schools are extensively covering this illness across the three domains. Furthermore, future research could involve an in-depth qualitative content analysis to determine more specific information regarding the amount and type of instruction, treatment, and research occurring in Medical Schools.

\section{Conclusion}

Overall, the current study suggests that there are a substantial number of schools that are not meeting one or all of the study criteria, suggesting that there is an extremely limited coverage of CFS at U.S. medical institutions. It is clear from the results of this study that CFS-related instruction, treatment, and especially research is at an unacceptably low level within the medical education community. This finding is supported by previous research revealing a gap in the degree of exposure in medical textbooks [10]. It is important for medical schools to devote more time and resources to research, education, and treatment of CFS, especially for the purpose of training future physicians to effectively recognize, diagnose, help find a cure for, and treat individuals suffering from this highly complex and often misunderstood illness.

\section{REFERENCES}

[1] K. Fukuda, S.E. Straus, I. Hickie, M.C. Sharpe, J.G. Dobbins, \& A. Komaroff. The chronic fatigue syndrome: a comprehensive approach to its definition and study. Annals of Internal Medicine, Vol.121, No.12, 953-959, 1994.

[2] B.M. Carruthers, A.K. Jain, K.L. De Meirleir, D.L. Peterson, N.G. Klimas, A.M. Lerner, A.C. Bested, P. Flor-Henry, P. Joshi, A.C.P. Poweles, J.A. Sherkey, M.I. van de Sande. Myalgic encephalomyelitis/chronic fatigue syndrome: clinical working case definition, diagnostic and treatment protocols. Journal of chronic fatigue syndrome, Vol.11, No.1, 7-115., 2003.

[3] B.M. Carruthers, M.I. van de Sande, K. L. De Meirleir, N.G. Klimas, G.Broderick, T. Mitchell, D. Staines, A.C.P. Powles, N.Speight,R.Vallings,L.Bateman,B.Baumgarten-Austrheim, D.S. Bell, N.Carlo-Stella, J. Chia, A. Darragh, D.Jo,D. Lewis, A.R.Light, S. Marshall-Gradisbik, I. Mena, J. A. Mikovits, K. Miwa, M. Murovska, M. L. Pall, \& S. Stevens. Myalgic encephalomyelitis: international consensus criteria, Journal of Internal Medicine, Vol.207, No.4, 327-338, 2011.

[4] W.J. Gunn, D.B. Connell, \& B. Randall. Epidemiology of chronic fatigue syndrome: the Centers for Disease Control study. In Ciba Foundation Symposium 173-Chronic Fatigue Syndrome (pp. 83-101). John Wiley \& Sons, Ltd, 1993.

[5] L. A. Jason, . J. A. Richman, A.W. Rademaker, K. M. Jordan, A.V. Plioplys, R.R. Taylor, W. McCready, C. Huang, S. Plioplys. A community-based study of chronic fatigue syndrome. Archives of Internal Medicine, Vol.159, No.18, 2129-2137, 1999.

[6] W.C Reeves, J.F Jones, E. Maloney, C. Heim, D.C. Hoaglin, R.S. Boneva1, M. Morrissey, \& R. Devlin. Prevalence of chronic fatigue syndrome in metropolitan, urban, and rural Georgia. Popul Health Metr, Vol.5, No.5, 1-10, 2007.

[7] M. Reyes, R. Nisenbaum, D.C. Hoaglin, E.R. Unger, C. Emmons, B. Randall, J.A. Stewart, S. Abbey, J. F. Jones, N. Gantz, S. Minden, W. C. Reeves.Prevalence and incidence of chronic fatigue syndrome in Wichita, Kansas. Archives of Internal Medicine, Vol.163, No.13, 1530, 2003.

[8] W.C. Reeves, D. Wagner, R. Nisenbaum, J.F. Jones, B. Gurbaxani, L.Solomon, D.A. Papanicolaou, E.R. Unger, S. D. Vernon, \& C. Heim.Chronic fatigue syndrome-a clinically empirical approach to its definition and study. BMC medicine, Vol.3, No.1, 19, 2005.

[9] L.A., Jason, N. Porter, A. Brown, M. Brown, \& M. Evans. A constructive debate with the CDC on the empirical case definition of chronic fatigue syndrome. Journal of Disability Policy Studies, Vol.20, No.4, 251-256, 2010.

[10] L.A. Jason, E. Paavola, N. Porter, \& M.L. Morello. Frequency and content analysis of chronic fatigue syndrome in medical text books. Australian journal of primary health, Vol.16, No.2, 174-178, 2010.

[11] M. W. Rabow, G. E. Hardie, J. M. Fair, \& S. J. McPhee. End-of-life care content in 50 textbooks from multiple specialties. JAMA: the journal of the American Medical Association, Vol.283, No.6, 771-778, 2007. 